\title{
Décomposition d'un critère énergétique : étude de l'endommagement dans les composites stratifiés croisés
}

\author{
JEAN-LUC REBIÈRE ${ }^{a}$ \\ Institut d'Acoustique et de Mécanique, Université du Maine, Avenue Olivier Messiaen, 72085 Le Mans Cedex 9, France
}

Reçu le 17 novembre 2004 ; accepté le 19 décembre 2005

\begin{abstract}
Résumé - Cette étude s'inscrit dans la suite de l'analyse du champ des contraintes dans des composites stratifiés croisés symétriques endommagés de type $\left[0_{\mathrm{m}}, 90_{\mathrm{n}}\right]_{\mathrm{s}}$ sollicités par un chargement uni-axial dans leur plan. Le résultat de la répartition des contraintes a conduit à la décomposition d'un critère énergétique en vue d'étudier la contribution des différentes composantes du tenseur des contraintes au taux de restitution d'énergie. À partir de cette approche, l'étude des mécanismes d'endommagements est présentée pour les différents modes de rupture. Cette approche doit contribuer à une meilleure compréhension des mécanismes d'endommagements par fissuration et par délaminage.
\end{abstract}

Mots clés : Fissure transverse / fissure longitudinale / délaminage / critère / décomposition du taux de restitution d'énergie

\begin{abstract}
A decomposition of an energetical criterion: study of damage growth in composites cross-ply laminates. This study follows the stress field analysis in a damaged symmetrical cross-ply laminate like $\left[0_{\mathrm{m}}, 90_{\mathrm{n}}\right]_{\mathrm{s}}$ by uni-axial loading in the plate plan. The stress distribution leads us to decompose a criterion. This criterion is based on the computation of the partial strain energy release rate associated with each damage mode, transverse cracking, longitudinal cracking and delamination. Several decompositions of the complementary strain energy are put forward. Each component part of this decomposition is related to a specific damage mechanism and loading mode. The related criterion can predict and describe the initiation and propagation of the different damage mechanisms.
\end{abstract}

Key words: Transverse cracking / longitudinal cracking / delamination / damage criterion / decomposition of the strain energy release rate

\section{Introduction}

Bien que les matériaux composites soient utilisés dans de nombreuses applications structurales pour leur gain en masse spécifique, leur tenue à long terme n'est pas encore toujours bien connue. Il est donc nécessaire d'utiliser des critères qui permettent de suivre de façon convenable l'évolution de l'endommagement dans ces stratifiés. Le premier endommagement observé dans les stratifiés croisés est généralement la fissuration transverse. Cet endommagement est suivi soit par la fissuration longitudinale soit par le délaminage. De nombreux critères ont été proposés dans la littérature pour étudier le développement des différents mécanismes d'endommagements dans les composites stratifiés croisés [1-13]. Ces critères sont généralement basés sur le calcul de la contrainte maximum. D'autres critères reposent sur des approches énergétiques. Dans [14,15], plusieurs modèles

\footnotetext{
a Auteur correspondant :

jean-luc.rebiere@univ-lemans.fr
}

analytiques et numériques ont été utilisés pour prédire uniquement l'amorçage de la fissuration transverse dans les stratifiés croisés.

Dans le présent papier, nous proposons un critère énergétique au sens de la mécanique linéaire de la rupture. L'apport principal de ce travail concerne l'utilisation de ce critère pour l'analyse de l'évolution de l'endommagement, et plus particulièrement l'estimation du calcul de certaines « parts » du taux de restitution d'énergie qui contribueront à une meilleure compréhension de ces phénomènes d'endommagements des stratifiés croisés. À l'aide de ce critère, l'estimation de l'amorçage des trois types d'endommagements et donc la contribution de chacun de ces mécanismes d'endommagement au taux de restitution d'énergie a été isolée. Ensuite et avec cette approche énergétique, une décomposition du taux de restitution d'énergie du stratifié complet est proposée en donnant la contribution des principaux mécanismes d'endommagement pour chaque mode de rupture (mode I, II ou III) (voir Tab. 1) [16]. Ces estimations des taux de 


\section{Nomenclature}

\begin{tabular}{|ll|}
\hline$a$ & demi-espace entre 2 fissures transverses \\
$A_{\mathrm{d}}$ & surface délaminée \\
$A_{\mathrm{f}}$ & surface fissurée \\
$b$ & demi-espace entre 2 fissures longitudinales \\
$d_{x}$ & longueur délaminée suivant la longueur $x$ \\
$d_{y}$ & longueur délaminée suivant la largeur $y$ \\
$\lambda$ & confinement \\
$G$ & taux de restitution d'énergie \\
$G_{\mathrm{FT}}$ & taux de restitution d'énergie associé à la fissuration transverse \\
$G_{\mathrm{FL}}$ & taux de restitution d'énergie associé à la fissuration longitudinale \\
$G_{\mathrm{d} x}, G_{\mathrm{d} y}$ & taux de restitution d'énergie associé au délaminage suivant $x$ ou suivant $y$ \\
$G_{\mathrm{C}}$ & taux de restitution d'énergie critique \\
$G_{\mathrm{Crf}}$ & taux de restitution d'énergie critique pour la fissuration \\
$G_{\mathrm{Crd}}$ & taux de restitution d'énergie critique pour le délaminage \\
$k$ & indice du pli \\
$L_{1}$ & longueur du stratifié suivant la direction $x$ \\
$L_{2}$ & longueur du stratifié suivant la direction $y$ \\
$M$ & nombre de fissures longitudinales, suivant la largeur $y$ \\
$N$ & nombre de fissures transverses, suivant la longueur $x$ \\
$t_{0}$ & épaisseur du pli à $0^{\circ}$ \\
$t_{90}$ & épaisseur du pli à $90^{\circ}$ \\
$U_{\mathrm{d}}$ & énergie de déformation du stratifié complet \\
$U_{\mathrm{cel}}$ & énergie de déformation de la cellule élémentaire \\
$U_{i j}$ & part de l'énergie de déformation relative à chaque contrainte \\
$V$ & volume de la cellule élémentaire \\
\hline & \\
\hline
\end{tabular}

restitution d'énergie sont associées à chaque composante du champ des contraintes pour déterminer l'initiation et la propagation des différents modes d'endommagements comme la fissuration transverse, la fissuration longitudinale et le délaminage. Cette décomposition est présentée pour chaque mode de rupture. De nombreuses simulations numériques ont permis d'estimer l'influence de chaque composante du tenseur des contraintes sur l'énergie de déformation. Dans le critère proposé, le taux de restitution d'énergie total est remplacé par des quantités qui sont reliées directement à un des mécanismes d'endommagement.

L'approche énergétique proposée, fondée sur la mécanique linéaire élastique de la rupture, est appliquée à l'ensemble des phénomènes d'endommagement par fissuration transverse et longitudinale ainsi que d'endommagement par délaminage. Ceci a été réalisé sans la prise en compte de l'interaction entre les différents mécanismes d'endommagement.

\section{Problème}

\subsection{Critère énergétique}

Le calcul du taux de restitution d'énergie a été réalisé à partir d'une approche variationnelle utilisée pour l'étude de la répartition du champ des contraintes dans les stratifiés endommagés. L'éprouvette étudiée est un stratifié croisé de type $\left[0_{\mathrm{m}}, 90_{\mathrm{n}}\right]_{\mathrm{s}}$ représenté sur la figure 1 . Les paramètres utilisés pour décrire l'architecture du stratifié sont : le confinement $(\lambda)\left(\lambda=t_{0} / t_{90}\right.$, où $t_{0}$ est l'épaisseur du pli à $0^{\circ}$ et $t_{90}$ est l'épaisseur du pli à $90^{\circ}$ ) et l'épaisseur de chacun des plis orientés à $0^{\circ}$ et à $90^{\circ}$. Le plan médian de la plaque stratifiée (plan xoy) est un plan de symétrie géométrique. Sur les stratifiés croisés de type $\left[0_{\mathrm{m}}, 90_{\mathrm{n}}\right]_{\mathrm{s}}$, les endommagements observés sont la rupture de la matrice entre les fibres nommée la fissuration et le délaminage qui correspond au décollement au niveau de l'interface entre les couches désorientées. Expérimentalement, la succession des endommagements suivants a été observée : la fissuration transverse apparaît en premier dans les plis désorientés par rapport à l'axe prédominant du chargement. Cet endommagement est suivi soit par la fissuration longitudinale soit par le délaminage. Dans cet article, le délaminage sera présenté sous une forme triangulaire à l'interface au niveau du croisement des fissures transverses et des fissures longitudinales.

La démarche suivie pour le calcul, avec cette approche fondée sur la mécanique linéaire élastique de la rupture, est la suivante. Pour évaluer le taux de restitution d'énergie, le stratifié est supposé préalablement endommagé. 


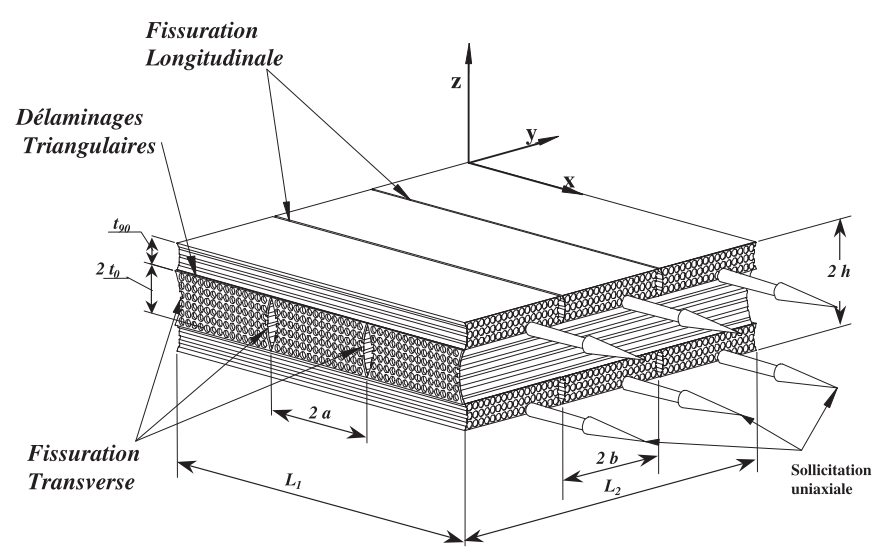

Fig. 1. Stratifié tri-couche endommagé par fissuration transverse, fissuration longitudinale et délaminage.

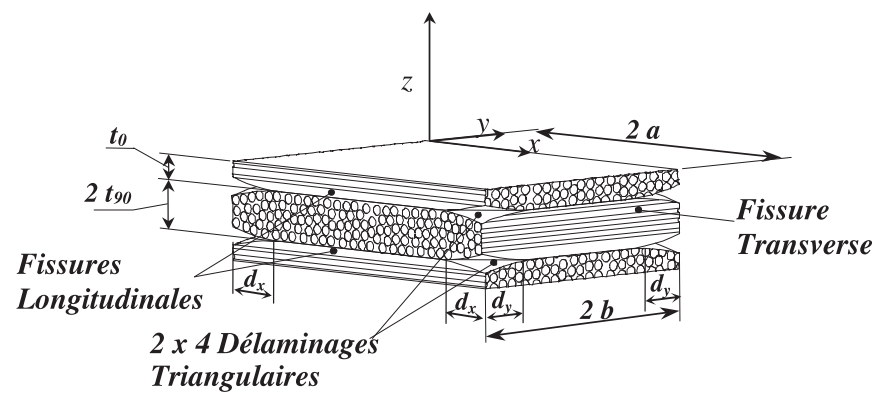

Fig. 2. Schématisation de la cellule élémentaire comprise entre 2 fissures transverses et longitudinales consécutives. Représentation des délaminages triangulaires à l'interface $0^{\circ} / 90^{\circ}$ en fonction de $d_{x}$ et $d_{y}$.

Par hypothèse, il existe des fissures transverses, des fissures longitudinales ainsi que des délaminages de formes triangulaires (Figs. 1 et 2). Les fissures sont supposées être de géométrie rectangulaire plane et chaque fissure s'étend sur toute l'épaisseur et toute la longueur du pli à 90 degrés endommagé. Des hypothèses géométriques similaires sont faites pour les fissures longitudinales dans les couches à 0 degré. La répartition des fissures est supposée uniforme suivant les deux directions $x$ et $y$. Ces hypothèses conduisent à l'étude de la cellule élémentaire représentée sur la figure 2. Cette cellule élémentaire est située entre deux fissures transverses consécutives et deux fissures longitudinales consécutives.

Le calcul du champ des contraintes, avec l'approche variationnelle proposée, est obtenu de la façon suivante [17] :

$$
\sigma_{i j}^{T(k)}=\sigma_{i j}^{0(k)}+\sigma_{i j}^{P(k)}
$$

Pour un stratifié croisé de type $\left[0_{\mathrm{m}}, 90_{\mathrm{n}}\right]_{\mathrm{s}}$ nonendommagé, sollicité par un chargement uni-axial suivant $x$ (Fig. 1), les contraintes sont notées $\sigma_{i j}^{0(k)}$. Elles sont obtenues à l'aide de la théorie des plaques stratifiées $\left(k=0^{\circ}, 90^{\circ}\right)$. La rupture de la matrice provoque des perturbations dans les plis à $0^{\circ}$ et $90^{\circ}$ notés $\sigma_{i j}^{P(k)}[17]$. Les délaminages locaux [18], de forme triangulaire, sont représentés sur la figure 2. Cette schématisation est confirmée par les résultats d'essais expérimentaux concernant le délaminage à l'interface $\left(0^{\circ} / 90^{\circ}\right)$ [1-13] et principalement au moment de l'amorçage de cet endommagement. Au niveau des interfaces, la surface non-endommagée par le délaminage est supposée n'être pertubée que par la fissuration. Dans la cellule élémentaire, au niveau de chaque interface $0^{\circ} / 90^{\circ}$, les quatre délaminages, de formes triangulaires, sont paramétrés par $d_{x}$ et $d_{y}$. Ces paramètres représentent les longueurs délaminées respectivement le long des fissures transverses et le long des fissures longitudinales. Dans les zones délaminées le frottement au niveau de l'interface et les efforts normaux suivant l'épaisseur sont négligés. Les paramètres $d_{x}$ et $d_{y}$ peuvent avoir des valeurs différentes. Par exemple pour schématiser un développement du délaminage le long des fissures transverses uniquement, les paramètres d'endommagement seraient les suivants : $d_{x} \neq d_{y}$ avec $d_{y} \approx 0$.

\subsection{Taux de restitution d'énergie}

Par définition le taux de restitution d'énergie $G$ associé à l'initiation et au développement de l'endommagement, pour un chargement donné, a pour expression :

$$
G=\frac{d}{d A} \widetilde{U}_{\mathrm{d}}(\sigma, A)
$$

où $\widetilde{U}_{\mathrm{d}}$ représente l'énergie de déformation du stratifié complet et $A$ l'étendue de la surface endommagée. $L_{1}$ représente la longueur de l'éprouvette suivant $x$ et $L_{2}$ la largeur suivant $y$. L'énergie de déformation dans la cellule élémentaire est notée $U_{\text {cel }}$. Les expressions de l'énergie de déformation $U_{\text {cel }}$, utilisées pour l'analyse du développement de la fissuration et du délaminage, sont développées dans [19].

Pour le stratifié complet, l'énergie de déformation a pour expression :

$$
\widetilde{U}_{\mathrm{d}}=N \cdot M \cdot U_{\text {cel }}
$$

où $N\left(N=L_{1} / 2 \bar{a} t_{90}\right)$ représente le nombre de fissures transverses et $M\left(M=L_{2} / 2 \bar{b} t_{90}\right)$ le nombre de fissures longitudinales. Considérons les quantités sans dimension suivantes : $\bar{x}=x / t_{90}, \bar{y}=y / t_{90}, \bar{z}=z / t_{90}, \bar{h}=h / t_{90}, \bar{a}=$ $a / t_{90}, \bar{b}=b / t_{90}, \overline{d_{x}}=d_{x} / t_{90}$ et $\overline{d_{y}}=d_{y} / t_{90}$. La densité de fissures transverses est définie par $d_{\mathrm{t}}\left(d_{\mathrm{t}}=1 / 2 a\right)$ et la densité de fissures longitudinales par $d_{\mathrm{l}}\left(d_{\mathrm{l}}=1 / 2 b\right)$. L'aire fissurée est représentée par $A_{\mathrm{f}}\left(A_{\mathrm{f}}=L_{1} L_{2}(1 / \bar{a}+\right.$ $1 / \bar{b}))$ et l'aire délaminée par $A_{\mathrm{d}}$ avec $\left(A_{\mathrm{d}}\left(\bar{d}_{x}, \bar{d}_{y}\right)=A_{\mathrm{d}}=\right.$ $\left.L_{1} L_{2}\left(\bar{d}_{x} \bar{d}_{y} / 2 \bar{a} \bar{b}\right)\right)$.

Les formules exposées ci-dessous permettent d'estimer la part du taux de restitution d'énergie relatif à chaque mécanisme d'endommagement. Le taux de restitution d'énergie associé à la fissuration transverse est noté $G_{\mathrm{FT}}$, celui associé à la fissuration longitudinale est noté $G_{\mathrm{FL}}$.

Les expressions de $G_{\mathrm{FT}}$ et de $G_{\mathrm{FL}}$ sont les suivantes :

$$
G_{\mathrm{FT}}=\frac{d \widetilde{U}_{\mathrm{d}}}{d A_{\mathrm{f}}}=\frac{d \widetilde{U}_{\mathrm{d}}}{d \bar{a}} \frac{d \bar{a}}{d A_{\mathrm{f}}} \quad G_{\mathrm{FL}}=\frac{d \widetilde{U}_{\mathrm{d}}}{d A_{\mathrm{f}}}=\frac{d \widetilde{U}_{\mathrm{d}}}{d \bar{b}} \frac{d \bar{b}}{d A_{\mathrm{f}}}
$$


Tableau 1. Composante des contraintes associées aux mécanismes d'endommagement pour les trois modes de rupture.

\begin{tabular}{lccc}
\hline & Fissuration transverse & Fissuration longitudinale & Délaminage \\
\hline Mode $I$ & $\sigma_{x x}^{(90)}$ & $\sigma_{y y}^{(0)}$ & $\sigma_{z}=\sigma_{z z}\left(z=t_{90}\right)$ \\
Mode II & $\sigma_{x z}^{(90)}$ & $\sigma_{y z}^{(0)}$ & $\tau_{x z}=\sigma_{x z}\left(z=t_{90}\right)$ \\
Mode III & $\sigma_{x y}^{(90)}$ & $\sigma_{x y}^{(0)}$ & $\tau_{y z}=\sigma_{y z}\left(z=t_{90}\right)$ \\
\hline
\end{tabular}

En utilisant les équations (3), (4) et les densités de fissures, les taux de restitution d'énergie associés à l'endommagement transverse $G_{\mathrm{FT}}$ et à l'endommagement longitudinal $G_{\mathrm{FL}}$ peuvent s'écrire alors sous la forme suivante :

$$
\begin{aligned}
G_{\mathrm{FT}} & =\frac{1}{2 \bar{b} t_{90}^{2}}\left(U_{\text {cel }}-\bar{a} \frac{d U_{\text {cel }}}{d \bar{a}}\right) \\
G_{\mathrm{FL}} & =\frac{1}{2 \bar{a} t_{90}^{2} \lambda}\left(U_{\text {cel }}-\bar{b} \frac{d U_{\text {cel }}}{d \bar{b}}\right)
\end{aligned}
$$

Les taux de restitution d'énergie, associés à l'initiation et à la propagation du délaminage suivant les directions $x$ et $y$, sont appelés respectivement $G_{d x}$ et $G_{d y}$ :

$$
G_{d_{x}}=\frac{d \widetilde{U}_{\mathrm{d}}}{d \bar{d}_{x}} \frac{d \bar{d}_{x}}{d A_{\mathrm{d}}} \quad G_{d_{y}}=\frac{d \widetilde{U}_{\mathrm{d}}}{d \bar{d}_{y}} \frac{d \bar{d}_{y}}{d A_{\mathrm{d}}}
$$

En utilisant les équations (3), (6) ainsi que les longueurs délaminées, les taux de restitution d'énergie associés à l'initiation et au développement de l'endommagement par délaminage (ici de forme triangulaire), suivant les directions $x$ et $y$, ont pour expressions :

$$
G_{d_{x}}=\frac{1}{2 \bar{d}_{y} t_{90}^{2}} \frac{d U_{\mathrm{cel}}}{d \bar{d}_{x}} \quad G_{d_{y}}=\frac{1}{2 \bar{d}_{x} t_{90}^{2}} \frac{d U_{\text {cel }}}{d \bar{d}_{y}}
$$

\subsection{Décomposition du taux de restitution d'énergie}

L'objectif principal de cette approche fondée sur la mécanique linéaire élastique de la rupture, est la décomposition du taux de restitution d'énergie qui consiste à proposer des approximations cohérentes des «parts » du taux de restitution d'énergie relative à chaque mécanisme d'endommagement pour les trois modes de rupture suivants : le mode $I$ (mode ouverture), le mode $I I$ (mode cisaillement) et le mode III (mode glissement) [16]. Dans le cas du stratifié croisé de type $\left[0_{\mathrm{m}}, 90_{\mathrm{n}}\right]_{\mathrm{s}}$, sollicité par un chargement uniaxial, le tableau 1 résume l'influence de chaque composante du tenseur des contraintes pour les trois modes de rupture précités. L'énergie de déformation (3) est décomposée pour obtenir la contribution de chaque produit deux à deux du champ des contraintes. Dans cet article, uniquement cinq de ces produits ont été retenus (9); après de nombreuses simulations numériques, nous avons observé que l'influence des autres produits restent très faibles ils sont donc négligés par hypothèse.

Le taux de restitution d'énergie du stratifié complet est défini dans l'équation (2). La contribution des composantes du tenseur des contraintes à l'énergie de déformation, dans les couches orientées à $0^{\circ}$ et à $90^{\circ}$ du stratifié endommagé, est donné par l'expression (8)

$$
\begin{aligned}
\widetilde{U}_{i j} & =2 . N \cdot M \cdot\left(U_{i j}^{90}+U_{i j}^{0}\right) \\
& =\frac{L_{1} L_{2}}{2 \bar{a} \bar{b} t_{90}^{2}}\left(U_{i j}^{90}+U_{i j}^{0}\right)=\frac{L_{1} L_{2}}{2 \bar{a} \bar{b} t_{90}^{2}} \cdot U_{i j}
\end{aligned}
$$

où les décompositions de l'énergie de déformation $U_{i j}$ sont définies par :

$$
\begin{aligned}
U_{x x}=U_{x x}^{90}+U_{x x}^{0}=\frac{1}{2 E_{\mathrm{T}}} \iiint_{V_{90}} \sigma_{x x}^{(90)^{2}} \mathrm{~d} \bar{x} \mathrm{~d} \bar{y} \mathrm{~d} \bar{z} \\
+\frac{1}{2 E_{\mathrm{L}}} \iiint_{V_{0}} \sigma_{x x}^{(0)^{2}} \mathrm{~d} \bar{x} \mathrm{~d} \bar{y} \mathrm{~d} \bar{z}
\end{aligned}
$$$$
U_{y y}=U_{y y}^{90}+U_{y y}^{0}=\frac{1}{2 E_{\mathrm{L}}} \iiint_{V_{90}} \sigma_{y y}^{(90)^{2}} \mathrm{~d} \bar{x} d \bar{y} \mathrm{~d} \bar{z}
$$$$
+\frac{1}{2 E_{\mathrm{T}}} \iiint_{V_{0}} \sigma_{y y}^{(0)^{2}} \mathrm{~d} \bar{x} \mathrm{~d} \bar{y} \mathrm{~d} \bar{z}
$$

$$
\begin{aligned}
U_{z z}=U_{z z}^{90}+U_{z z}^{0}= & \frac{1}{2 G_{\mathrm{LT}}} \iiint_{V_{90}} \sigma_{z z}^{(90)^{2}} \mathrm{~d} \bar{x} \mathrm{~d} \bar{y} \mathrm{~d} \bar{z} \\
& +\frac{1}{2 G_{\mathrm{TT}}} \iiint V_{0} \sigma_{z z}^{(0)^{2}} \mathrm{~d} \bar{x} \mathrm{~d} \bar{y} \mathrm{~d} \bar{z}
\end{aligned}
$$

$$
\begin{aligned}
& U_{x z}=U_{x z}^{90}+U_{x z}^{0}=\frac{1}{2 G_{\mathrm{TT}^{\prime}}} \iiint_{V_{90}} \sigma_{x z}^{(90)^{2}} \mathrm{~d} \bar{x} \mathrm{~d} \bar{y} \mathrm{~d} \bar{z} \\
&+\frac{1}{2 G_{\mathrm{LT}}} \iiint_{V_{0}} \sigma_{x z}^{(0)^{2}} \mathrm{~d} \bar{x} \mathrm{~d} \bar{y} \mathrm{~d} \bar{z} \\
& U_{y z}=U_{y z}^{90}+U_{y z}^{0}=\frac{1}{2 G_{\mathrm{LT}}} \iiint_{V_{90}} \sigma_{y z}^{(90)^{2}} \mathrm{~d} \bar{x} \mathrm{~d} \bar{y} \mathrm{~d} \bar{z} \\
&+\frac{1}{2 G_{\mathrm{TT}}} \iiint_{V_{0}} \sigma_{y z z}^{(0)^{2}} \mathrm{~d} \bar{x} \mathrm{~d} \bar{y} \mathrm{~d} \bar{z}
\end{aligned}
$$

dans la cellule élémentaire $V_{0}$ représente le volume du demi-pli à $0^{\circ}\left(|x| \leq a,|y| \leq b\right.$ et $\left.t_{90} \leq|z| \leq h\right)$ et $V_{90}$ le volume du demi-pli à $90^{\circ}\left(|x| \leq a,|y| \leq b\right.$ et $\left.|z| \leq t_{90}\right)$.

La contribution du produit des composantes des contraintes au taux de restitution d'énergie est obtenue à partir de la relation suivante :

$$
G_{i j}^{k}=\frac{d \widetilde{U}_{i j}^{k}}{d A} \quad \text { où } i=x, y, z \text { et } k=0^{\circ}, 90^{\circ}
$$


Tableau 2. Propriétés mécaniques et géométriques du pli d'un carbone/époxy T300/914 [20].

\begin{tabular}{cc}
\hline & Carbone/Époxy \\
\hline$E_{\mathrm{LT}}(\mathrm{GPa})$ & 140 \\
$E_{\mathrm{TT}^{\prime}}(\mathrm{GPa})$ & 10 \\
$G_{\mathrm{LT}}(\mathrm{GPa})$ & 5,7 \\
$G_{\mathrm{TT}^{\prime}}(\mathrm{GPa})$ & 3,6 \\
$\nu_{\mathrm{LT}}$ & 0,31 \\
$\nu_{\mathrm{TT}}$ & 0,48 \\
Épaisseur pli $(\mathrm{mm})$ & 0,125 \\
$G_{\mathrm{crf}}\left(\mathrm{J} / \mathrm{m}^{2}\right)$ & 228 \\
$G_{\mathrm{crd}}\left(\mathrm{J} / \mathrm{m}^{2}\right)$ & 158 \\
\hline
\end{tabular}

Dans les travaux $[14,15]$, le taux de restitution d'énergie est décomposé en deux parties relatives $(G=$ $\left.G^{0}+G^{90}\right)$ qui représentent respectivement la contribution des plis orientés à $0^{\circ}$ et des plis orientés à $90^{\circ}$. Ces résultats ont été obtenus à l'aide de six modèles analytiques ainsi qu'un calcul par éléments finis avec SAMCEF $[14,15]$. Ces travaux concernaient l'endommagement par fissuration transverse uniquement, ils ont été utilisés pour l'analyse de l'influence de certaines hypothèses simplificatrices rencontrées dans de nombreux modèles analytiques et numériques de la littérature.

Dans le présent article, nous proposons d'estimer maintenant l'initiation et l'évolution des trois mécanismes d'endommagement suivants : fissuration transverse, fissuration longitudinale et délaminage local en pointe de fissure. Le calcul du taux de restitution d'énergie relatif aux composantes du champ des contraintes est obtenu à partir des relations (9). L'évolution des mécanismes d'endommagement est estimée pour chaque mode de rupture à l'aide du tableau 2. La démarche proposée permet ainsi de remplacer le taux de restitution d'énergie global du stratifié par les approximations des «parts » du taux de restitution d'énergie attribuable à chaque mécanisme d'endommagement proprement dit.

L'approximation du taux de restitution d'énergie, associé à l'endommagement par fissuration transverse pour les deux modes de chargement (mode $I$ et mode $I I$ ) peuvent s'obtenir avec les relations suivantes

$$
G_{I}^{\mathrm{FT}}=\frac{d \widetilde{U}_{x x}}{d \bar{a}} \frac{d \bar{a}}{d A_{\mathrm{f}}} \quad G_{I I}^{\mathrm{FT}}=\frac{d \widetilde{U}_{x z}}{d \bar{a}} \frac{d \bar{a}}{d A_{\mathrm{f}}}
$$

L'approximation du taux de restitution d'énergie, associée à l'endommagement par fissuration longitudinale pour les deux modes de chargement (mode I et mode II) s'obtiennent à partir des relations suivantes

$$
G_{I}^{\mathrm{FL}}=\frac{d \widetilde{U}_{y y}}{d \bar{b}} \frac{d \bar{b}}{d A_{\mathrm{f}}} \quad G_{I I}^{\mathrm{FL}}=\frac{d \widetilde{U}_{y z}}{d \bar{b}} \frac{d \bar{b}}{d A_{\mathrm{f}}}
$$

L'approximation du taux de restitution d'énergie, associée à l'endommagement par délaminage pour les trois modes de chargement (mode I, mode II et mode III) ont pour expressions :

$$
G_{I}^{\mathrm{Del}}=\frac{d \widetilde{U}_{z z}}{d A_{\mathrm{d}}} \quad G_{I I}^{\mathrm{Del}}=\frac{d \widetilde{U}_{x z}}{d A_{\mathrm{d}}} \quad G_{I I I}^{\mathrm{Del}}=\frac{d \widetilde{U}_{y z}}{d A_{\mathrm{d}}}
$$

Ces expressions du taux de restitution d'énergie (13) associées à l'endommagement par délaminage correspondent ici au cas où la longueur délaminée est la même suivant les directions $x$ et $y$, c'est-à-dire le cas où $d_{x}=d_{y}$.

Toutes les décompositions du taux de restitution d'énergie, associées à chaque mécanisme d'endommagement et pour chaque mode de rupture (11)-(13), sont ainsi déterminées. Ainsi, l'évolution des différents mécanismes d'endommagements est obtenue à l'aide du critère d'endommagement (14). Pour chacune des quantités (11)-(13), les résultats sont confrontés aux valeurs critiques du taux de restitution d'énergie :

$$
G=G_{\mathrm{c}}
$$

Le calcul du taux de restitution d'énergie associé à chaque mécanisme d'endommagement est réalisé de la façon suivante. Par exemple, pour l'endommagement par fissuration transverse, le taux de restitution d'énergie total est remplacé par une quantité approximative relative au mode $I$ et au mode II. Les deux valeurs ainsi calculées sont comparées avec des valeurs critiques $G_{\text {crf }}$ (valeur de $\left.G_{\text {crf }}[20]\right)$. Ce calcul permet d'estimer, à l'aide de notre approche, si l'amorçage de la fissuration transverse s'opère en mode ouverture ou en mode cisaillement.

Pour l'amorçage et le développement des autres mécanismes d'endommagements (par fissuration longitudinale ou par délaminage), une densité de fissure transverse est alors fixée pour les calculs. Le choix de ces densités de fissures découlent principalement de l'analyse de la saturation de l'endommagement par fissuration transverse $[15,17]$.

Pour chacune de ces valeurs, toutes les décompositions partielles du taux de restitution d'énergie sont évaluées (11)-(13) et comparées aux valeurs critiques $G_{\text {crf }}$ et $G_{\text {crd }}(14)$. Il en est de même pour l'amorçage du délaminage, les paramètres $d_{x}$ et $d_{y}$ tendent vers zéro pour des densités d'endommagements transverses et longitudinaux fixés.

\section{Résultats}

Les résultats proposés concernent la décomposition du taux de restitution d'énergie associé aux trois mécanismes d'endommagement et ceci pour tous les modes de rupture. Les décompositions, développées au paragraphe précédent, permettent d'étudier l'influence de l'architecture (rapport des épaisseurs des couches) de la plaque stratifiée à plis croisés de type $\left[0_{\mathrm{m}}, 90_{\mathrm{n}}\right]_{\mathrm{S}}$ sur l'endommagement par fissuration transverse, fissuration longitudinale et délaminage. Le matériau étudié est un carbone époxy T300-914. Les paramètres de l'étude sont l'épaisseur des couches à $0^{\circ}$ et à $90^{\circ}$. Dans tous les résultats exposés, 


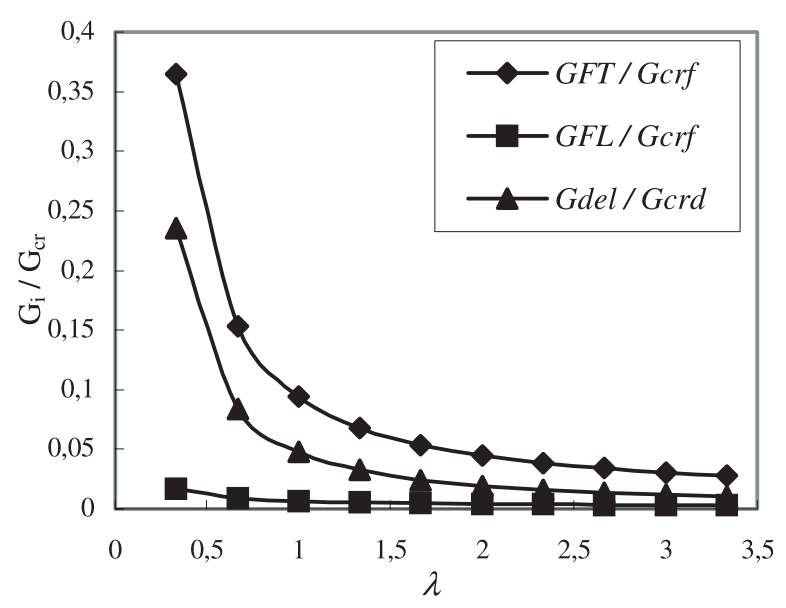

Fig. 3. Variation du taux de restitution d'énergie associé à l'initiation de la fissuration transverse $\left(G_{\mathrm{FT}}\right)$, de la fissuration longitudinale $\left(G_{\mathrm{FL}}\right)$ et du délaminage $\left(G_{\mathrm{del}}\right)$ en fonction du confinement $\lambda$. Le stratifié en carbone époxy est composé de 8 plis.

les simulations numériques ont été réalisées pour un chargement uni-axial suivant $x$ de $150 \mathrm{MPa}$. Toutes ces décompositions du taux de restitution d'énergie, associées à l'endommagement par fissuration transverse, fissuration longitudinale et délaminage, sont normalisées par les valeurs critiques du taux de restitution d'énergie appelé $G_{\mathrm{crf}}$ pour la fissuration et $G_{\text {crd }}$ pour le délaminage.

Sur la figure 3, l'initiation de l'endommagement est suivie à partir du « paramètre d'endommagement $a / t_{90}$ ». L'amorçage est caractérisé par un 1/2 espacement entre deux fissures consécutives « $a »$ ( paramétré par $\left.a / t_{90}\right)$ qui tend vers l'infini. Numériquement dans les travaux [15], les résultats, du taux de restitution d'énergie associé à l'initiation de l'endommagement transverse, restent stables [16] pour des valeurs du paramètre d'endommagement « $a / t_{90} »$ de l'ordre de 8 . La valeur de ce paramètre d'endommagement a donc été retenue pour la suite des travaux exposés.

Les résultats présentés sur la figure 3 montrent que pour toutes les valeurs du confinement $\lambda$ (quelle que soit l'épaisseur des plis du stratifié tri-couche), la valeur du taux de restitution d'énergie $G_{\mathrm{FT}}\left(G_{\mathrm{FT}}\right.$ évalué avec l'équation (5)) associée à la fissuration transverse est toujours la plus importante. Pour ce stratifié de 8 plis, la fissuration transverse est bien le premier type d'endommagement observé. De plus, les taux de restitution d'énergie associés aux autres mécanismes d'endommagement $G_{\mathrm{FL}}$ ou $G_{\mathrm{del}}\left(G_{\mathrm{FL}}\right.$ ou $G_{\mathrm{del}}$ évalués avec les équations $\left.(6),(7)\right)$, suivent les mêmes lois de variation. Sur toutes les courbes proposées, les simulations numériques, du taux de restitution d'énergie en fonction du paramètre confinement $(\lambda)$, sont des fonctions décroissantes. Ceci confirme bien que l'initiation de l'endommagement, par fissuration transverse, fissuration longitudinale ou délaminage soit plus difficile dans le cas d'un stratifié contenant une couche à $0^{\circ}$ épaisse. Pour le stratifié de 8 plis, quand le confinement $(\lambda)$ augmente, l'épaisseur des couches à $0^{\circ}$ devient plus importante. Ainsi les fibres orientées à $0^{\circ}$ supportent la

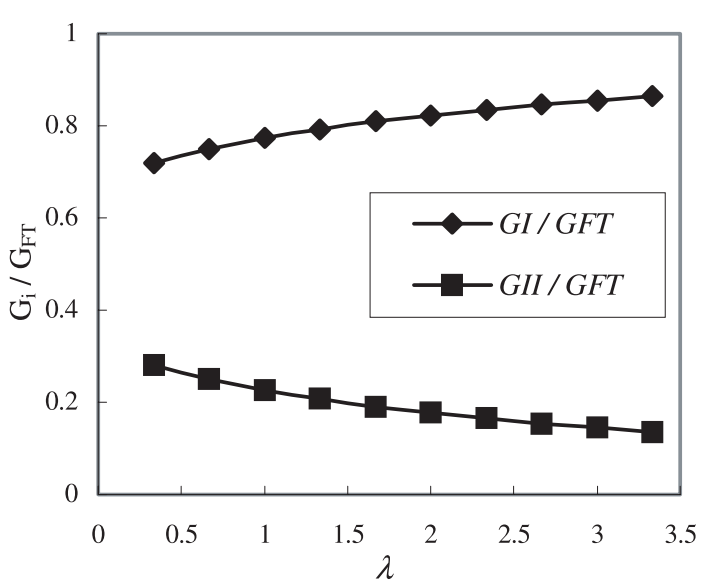

Fig. 4. Initiation de la fissuration transverse dans un stratifié de 8 plis en carbone époxy. Modes de rupture $G_{I} / G_{\mathrm{FT}}$, $G_{I I} / G_{\mathrm{FT}}$ en fonction du confinement $\lambda$.

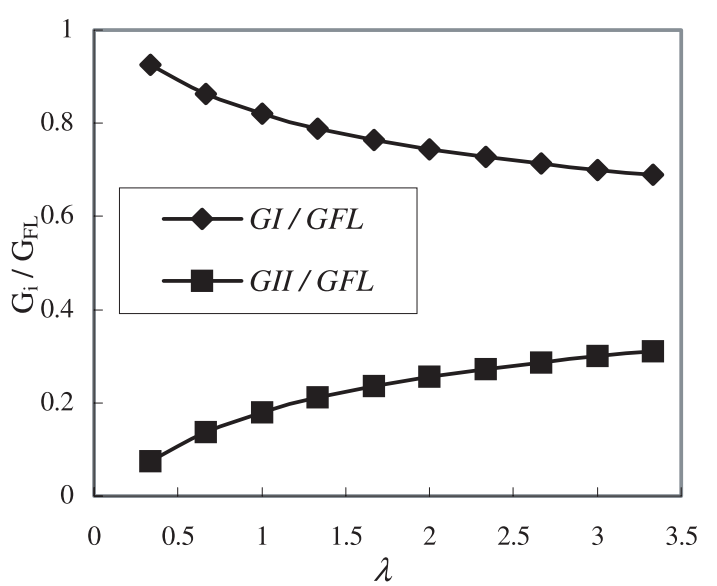

Fig. 5. Initiation de la fissuration longitudinale dans un stratifié de 8 plis en carbone époxy. Modes de rupture $G_{I} / G_{\mathrm{FL}}$, $G_{I I} / G_{\mathrm{FL}}$ en fonction du confinement $\lambda$.

majorité du chargement, ce qui retarde l'initiation de tous les mécanismes d'endommagements. Les conclusions des essais expérimentaux [20-24], sur l'influence de l'épaisseur de la couche à $0^{\circ}$ sur l'amorçage des différents mécanismes d'endommagements, rejoignent l'analyse de nos résultats.

La décomposition du taux de restitution d'énergie, associé à l'initiation de l'endommagement par fissuration transverse, fissuration longitudinale ou délaminage est présentée pour les différents modes de rupture (mode $I$, mode II et mode III). Sur les figures 4 et 5 , les résultats montrent que l'endommagement par fissuration transverse et par fissuration longitudinale est toujours initié en mode ouverture (mode $I$ ). C'est principalement la contrainte normale $\sigma_{x x}^{(90)}$ qui contrôle la propagation de la fissuration transverse. Pour la fissuration longitudinale c'est la contrainte normale $\sigma_{y y}^{(0)}$ qui pilote le phénomène.

Pour l'initiation du délaminage, c'est le confinement $(\lambda)$ qui gouverne le mode de rupture (Fig. 6). Nous pouvons observer que le délaminage est toujours créé en mode $I I$ pour des valeurs de $\lambda$ inférieures à l'unité $(\lambda<1)$. Ce mode de rupture est principalement gouverné par la 


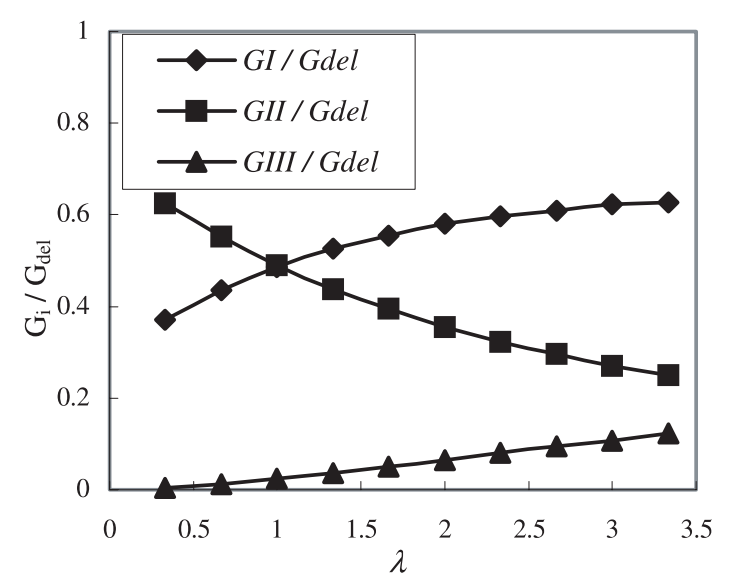

Fig. 6. Initiation du délaminage d'un stratifié de 8 plis en carbone époxy. Modes de rupture $G_{I} / G_{\mathrm{del}}, G_{I I} / G_{\mathrm{del}}, G_{I I I} / G_{\mathrm{del}}$ en fonction du confinement $\lambda$.

composante du cisaillement $\sigma_{x z}$ au niveau de l'interface des plis désorientés. Pour un confinement égal à l'unité $(\lambda=1)$, l'initiation du délaminage devient plus difficile. Ensuite, pour des valeurs du confinement $(\lambda)$ supérieures à l'unité $(\lambda>1)$, l'initiation se produit alors en mode $I$. Dans ce cas, c'est la contrainte normale suivant l'épaisseur de la plaque stratifiée, au niveau de l'interface qui contrôle alors le phénomène. Ceci a été confirmé par une étude analytique et par éléments finis [17,20].

\section{Conclusion}

Dans cet article, un critère énergétique au sens de la mécanique linéaire de la rupture est proposé. Les résultats des simulations numériques concernent des approximations de l'estimation de taux de restitution d'énergie associés à différents mécanismes d'endommagements pour les trois modes de rupture. La démarche proposée est, actuellement, l'aboutissement de travaux réalisés avec des approches analytiques et numériques.

Les courbes proposées sont les résultats de simulations numériques. Bien qu'aucun résultat expérimental ne soit reporté sur ces courbes, les tendances de ces courbes nous permettent de dresser cependant les conclusions suivantes.

Les résultats des simulations numériques confirment que l'endommagement par fissuration transverse est bien le premier mode d'endommagement observé.

De plus, si l'épaisseur de la couche à $90^{\circ}$ est importante, alors l'amorçage des trois modes d'endommagements sera facilité.

Dans tous les résultats proposés, l'amorçage de l'endommagement par fissuration transverse et par fissuration longitudinale est toujours initié en mode $I$.

Par contre pour l'amorçage du délaminage nous observons plusieurs cas avec ces simulations numériques. Dans les stratifiés peu confinés, le délaminage est initié en mode II. Lorsque le confinement $(\lambda)$ est supérieur à 1 , l'initiation du délaminage se fait alors en mode $I$. Il n'a pas été relevé de cas où l'initiation du délaminage se produit en mode III.

\section{Références}

[1] J.-C. Brewer, P.A. Lagace, Quadratic stress criterion for initiation of delamination, J. Compos. Mater. 22 (1988) $1141-1155$

[2] N.-V. Akshantala, R. Talreja, A mechanistic model for fatigue damage evolution in composite laminate, Mech. of Mater. 29 (1998) 123-140

[3] N.-V. Akshantala, R. Talreja, A micro mechanics model for predicting fatigue life of composite laminate, Mater. Sci. Eng. A 285 (2000) 303-313

[4] A.B. Pereira, A. de Morais, A.T. Marques, P.T. de Castro, Mode II interlaminar fracture of carbon/epoxy cross-ply multidirectional laminates, Comp. Sci. Technol. 62 (2002) 679-686

[5] C. Li, F. Ellyin, A. Warmby, On matrix crack saturation in composite laminates, Comp. Part B: Eng. 34 (2003) 473-480

[6] C.M. Dakshina Moorthy, J.N. Reddy, Recovery of interlaminar stresses and strain energy release rate in composite laminates. Finite Elem. in Analy. Design. 33 (1999) 1-27

[7] W. Yan, H.-Y. Liu, Y.-W. Mai, Numerical study on the mode I delamination toughness of the $\mathrm{Z}$ pinned laminates, Comp. Sci. Technol. 63 (2003) 1481-1493

[8] M.J. Hinton, A.S. Kaddour, P.D. Soden, Evaluation of failure prediction in composite laminates, Comp. Sci. Technol. 64 (2004) 321-327

[9] J.S. Mayes, A.C. Hansen, A comparison of multicontinuum theory based failure simulation with experimental results, Comp. Sci. Technol. 64 (2004) 517-527

[10] J.S. Mayes, A.C. Hansen, Composite laminate failure analysis using multicontinuum theory, Comp. Sci. Technol. 64 (2004) 379-394

[11] K.I. Tserpes, P. Papanikos, G. Labeas, Pantelakis Sp., Fatigue damage accumulation and residual strength assessment of CFRP laminates, Comp. Struct. 63 (2004) 219-230

[12] Z. Xia, Y. Chen, F. Ellyin, A meso/micro mechanical model for damage progression in glass fiber/epoxy cross-ply laminates by finite-element analysis, Comp. Sci. Technol. 60 (2000) 1171-1179

[13] D. Leguillon, A method based on singularity theory to predict edge delamination of laminates, Int. J. Fract. 100 (1999) 105-120

[14] D. Gamby, J. Martins, S. Maison, J.-L. Rebière, Influence du confinement sur le Taux de restitution d'énergie associé à la Fissuration Transverse d'un stratifié. Comparaison de deux simulations Numériques, in G. Fantozzi, P. Fleishman (éd.), Proceedings des Septièmes Journées Nationales sur les Matériaux Composites, Lyon, France, AMAC (1990) 609-617

[15] J.-L. Rebière, Modélisation du champ des contraintes créé par des fissures de fatigue dans un composite stratifié carbone/polymère, thèse de doctorat, Université de Poitiers, France, 1992

[16] J. Lemaître, J.-L. Chaboche, Mécanique des matériaux solides, Dunod $2^{\mathrm{e}}$ édition, 1988 
[17] J.-L. Rebière, M.-N. Maâtallah, D. Gamby, Initiation and growth of transverse and longitudinal cracks in composite cross-ply laminates, Comp. Struct. 53 (2001) 173-187

[18] J.-L. Rebière, M.-N. Maâtallah, D. Gamby, Analysis of damage mode evolution in a cross-ply laminate under uni axial loading, Comp. Struct. 55 (2002) 115-126

[19] J.-L. Rebière, D. Gamby, A criterion for modelling initiation and propagation of matrix cracking and delamination in cross-ply laminates, Comp. Sci. Technol. 64 (2004) 2239-2250

[20] A.S.D. Wang, F.W. Crossman, Initiation and growth of transverse cracks and edge delamination in composite laminates. Part I: An energy method, J. Comp. Mater. 14 (1980) 71-87
[21] A.S.D. Wang, N.M. Kishore, C.A. Li, Crack development in graphite-epoxy cross-ply laminates under uniaxial tension, Comp. Sci. Technol. 24 (1985) 1-31

[22] D.H. Allen, S.E. Groves, C.E. Harris, A thermomechanical constitutive theory for elastic composites with distributed damage, I. Theoretical development, Int. J. Solids Struct. 23 (1987) 1301-1318

[23] R.P. Harrison, M.G. Bader, Damage development in CFRP laminates under monotonic and cyclic stressing, Fibre Sci. Technol. 18 (1983) 163-180

[24] A.S.D. Wang, N.M. Kishore, Crack development in graphite-epoxy cross-ply laminates under uniaxial tension, Comp. Sci. Technol. 24 (1985) 1-31 